\title{
Photoelectron angular distribution of atoms in pulsed XUV and IR fields
}

\author{
Xiao-Min Tong ${ }^{1, \text { 米 }}$ \\ ${ }^{1}$ Center for Computational Sciences, University of Tsukuba, \\ 1-1-1 Tennodai, Tsukuba, Ibaraki 305-8573, Japan
}

\begin{abstract}
We investigate the photoelectron angular distribution of atoms in pulsed XUV and IR laser fields theoretically by solving the time-dependent Schrödinger equation and surprisingly find that the angular distributions of sidebands are not always straight lines (normal) in the energy-angle plot as predicted by the strong field approximation. Comparing our results with those of the strong field approximation, in which photoelectron nucleus Coulomb interaction is ignored, the bending of angular distribution is attributed to the photoelectron-nucleus Coulomb interaction. The bending depends on the IR intensity, IR pulse duration, XUV photon energy as well as the time-delay between the two pulses.
\end{abstract}

PACS numbers: $32.80 . \mathrm{Fb}, 32.80 . \mathrm{Rm}, 42.65 . \mathrm{Sf}$

\section{INTRODUCTION}

Combination of an extreme-ultraviolet (XUV) source generated from the high-order-harmonic generation (HHG) 1, 2] and the driving infrared (IR) laser opens a new door to investigate and control atomic photoabsorption processes. Several sidebands as predicted in theory [3] have been observed in experiments [4, 5]. If short XUV and IR pulses are used, the strengths of the sidebands can be controlled by the time-delay between the two pulses [6, 7.

The mechanism of the IR assisted photoabsorption can be explained as follows: the energy structures of an atom in an IR field are described by Floquet states [8, a Floquet state has many sidebands separated by one IR photon energy; and an electron can be ionized/excited by an XUV to a Floquet state through different sidebands, or different paths. Therefore, the energy distributions of photoelectron are also separated by one IR photon energy (sideband structure) and the sideband yields are sensitive to the relative phase or arriving time between the XUV and IR pulses [9, 10]. Therefore, one can control the sideband yields by steering the time-delay between the two pulses. Meanwhile, if one can control the relative strengths of the HHGs, one can also control the XUV photoabsorption cross-section as predicted in theory [11] and confirmed by experiment 12 .

Different from the XUV source generated from HHG, which is of the form of an attosecond pulse train $(\mathrm{APT})$ 13, 14, or a single attosecond pulse (SAP) 15, 16, the photon energy of the XUV source radiated from a free-electron laser [17] can be tuned almost continuously and the pulse width can be narrowed in a femto-second time-scale, so it opens another dimension to study the IR assisted photoabsorption process as recently reported in both theories [18, 19] and experiments [20, 21]. The

\footnotetext{
* tong.xiaomin.ga@u.tsukuba.ac.jp
}

two experiments focused on the photoelectron angular distribution.

When we investigated the photoelectron angular distribution of Ar atoms ionized by an XUV assisted with IR fields as reported recently in two experiments [20, 21, we surprisingly found that the sideband angular distributions are not iso-energy separated by one IR photon energy, or straight lines in energy-angle plots as predicted by the strong field approximation (SFA) $22-25$ if we use a lower XUV photon energy. We define this non-straightline distribution as bending of photoelectron angular distribution or simply bending in the following discussion.

To investigate the origin of the bending of photoelectron angular distribution and simplify the problem, we simulated the photoelectron angular distribution of $\mathrm{H}$ atoms in two-color XUV and IR laser pulses and confirmed that the angular distribution is not always isoenergy. Simply switching off the photoelectron nucleus Coulomb interaction in the simulation, the SFA does predict iso-energy distributions as shown in Fig. 1. which are in consistent with our present knowledge of the angular distribution for a multi-photon absorption. This clearly shows that the bending is attributed to the Coulomb effect which is ignored in the SFA.

In this work, we systematically investigated how the bending depends on the IR intensity, pulse duration and the time-delay between the two pulses. Since we focused on the IR laser assisted photoelectric effect, we assumed that the IR pulse is longer than the XUV pulse and both pulses are longer than one period of the IR laser in this paper.

\section{THEORETICAL METHOD}

The atomic photoabsorption by an XUV light in an IR field can be studied by solving the time-dependent Schrödinger equation (TDSE) numerically. The detailed method has been published [26] so we only brief the key working equations for the later discussion. To compare 


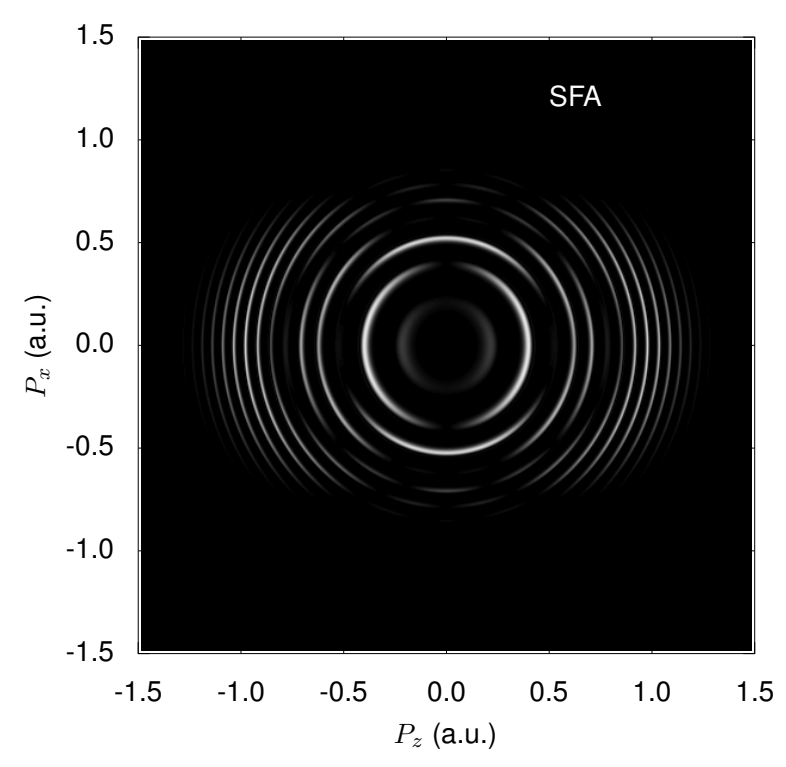

FIG. 1. Photoelectron momentum distribution of $\mathrm{H}$ atoms ionized by an XUV light in an IR field calculated by the SFA. The IR laser intensity is $2 \times 10^{13} \mathrm{~W} / \mathrm{cm}^{2}$.

with the SFA, we solve the TDSE in the integral form [27, 28. All the dynamical information can be obtained from the following equation [27] (atomic units, $\hbar=e=$ $m_{e}=1$, are used hereafter unless otherwise stated)

$$
\begin{aligned}
\Psi(t)= & -i \int_{-\infty}^{t} T\left(e^{-i \int_{t^{\prime \prime}}^{t} H\left(t^{\prime}\right) d t^{\prime}}\right) V_{X U V}\left(t^{\prime \prime}\right) e^{-i H_{0} t^{\prime \prime}} \Psi_{0} d t^{\prime \prime} \\
& +e^{-i H_{0} t} \Psi_{0} .
\end{aligned}
$$

Here, $T$ is the time ordering operator, $\Psi_{0}$ and $\Psi(t=$ $\infty)$ are the initial and final electron wave functions, $H(t)=H_{0}+V_{\text {ext }}(t)$ is the Hamiltonian of hydrogen atoms in an external field including the contributions of IR $\left(V_{I R}(t)\right)$ and the XUV $\left(V_{X U V}(t)\right)$ fields with $H_{0}$ being the external-field-free hydrogen Hamiltonian as

$$
H_{0}=-\frac{\nabla^{2}}{2}-\frac{1}{r}
$$

and

$$
V_{\text {ext }}(t)= \begin{cases}\mathbf{r} \cdot \mathbf{E}(t), & \text { length gauge } \\ \mathbf{p} \cdot \mathbf{A}(t)+\frac{A^{2}(t)}{2}, & \text { velocity gauge }\end{cases}
$$

being atom-laser interaction. The vector potential of the $\mathrm{XUV}$ is written as

$$
\mathbf{A}_{x}(t)=\frac{\mathbf{E}_{x}}{\omega_{x}} e^{-t^{2} / \tau_{x}^{2} 2 \ln 2} \sin \left(\omega_{x} t\right),
$$

where $\mathbf{E}_{x}, \omega_{x}$ and $\tau_{x}$ are the XUV electric field strength, center photon energy and the pulse duration of the full width at half magnitude, respectively. Similarly, the vector potential of the IR laser is written as

$$
\mathbf{A}_{\mathrm{IR}}(t)=\frac{\mathbf{E}_{0}}{\omega} e^{-\left(t+t_{d}\right)^{2} / \tau^{2} 2 \ln 2} \sin \left(\omega\left(t+t_{d}\right)+\delta\right),
$$

where $\mathbf{E}_{0}, \omega, \tau$ and $\delta$ are the electric field strength, photon energy, pulse duration, and the carrier-envelop-phase (CEP) of the IR pulse, respectively. $t_{d}$ is the time-delay between the two pulses and a positive $t_{d}$ means that the IR pulse arrives earlier. The total vector potential $\mathbf{A}(t)=\mathbf{A}_{x}(t)+\mathbf{A}_{\mathrm{IR}}(t)$ and the corresponding electric field is

$$
\mathbf{E}(t)=-\frac{d \mathbf{A}(t)}{d t}
$$

If we ignore the electron nucleus Coulomb interaction in Hamiltonian $H$ [see Eq. [1] ] in the velocity gauge (as shown in Eq. (3)), Eq. (1) goes to the SFA. In the following simulations, we assumed that the polarizations of the XUV and IR fields are parallel to each other as used in the experiments [20, 21] and we set the polarization direction as the $z$-direction.

\section{RESULTS AND DISCUSSION}

In this section, we mainly discuss the photoelectron angular distribution of $\mathrm{H}$ atoms ionized by an XUV light with $\omega_{x}=20 \mathrm{eV}$ photon energy and $\tau_{x}=8$ fs pulse duration in an IR field with $800 \mathrm{~nm}$ wavelength and $\tau=30$ fs pulse duration. In the simulation we fixed the XUV intensity of $10^{11} \mathrm{~W} / \mathrm{cm}^{2}$ so the high-order effect of XUV light is negligible small and the IR intensity is less than $50 I_{0}$ with $I_{0}=10^{12} \mathrm{~W} / \mathrm{cm}^{2}$ so the direct ionization by the IR laser is eliminated. We also present the results with other XUV and IR parameters to show how the bending depends on the XUV and IR laser parameters. Since we focused on the photoelectron angular distribution, we plotted the photoelectron yield as a function of the electron energy and the angle of the momentum to the $z$-direction instead of the momentum distribution as shown in Fig. 1 .

\section{A. Results of SFA and TDSE}

Figure 2 shows the typical photoelectron energy-angle distributions obtained by the SFA (upper panel) and TDSE (lower panel) simulations with the IR intensity of $20 I_{0}$. In the figure, the SFA predicts straight line distributions or iso-energy distributions (upper-panel in Fig. 2) while the TDSE shows that the sideband angular distributions in the energy-angle plots bend to higher energies for larger angles, especially for lower energy sidebands (lower-panel in Fig. 2). Since the SFA ignores the Coulomb interaction after the electron is released by the XUV, the bending must be related to the electron nucleus Coulomb interaction. Without IR fields, the photoelectron shows straight line distributions, so the bending is also coupled to the pulsed IR field. Since the bending depends on the XUV and IR laser parameters we will investigate how the parameters affect the angular distributions. 

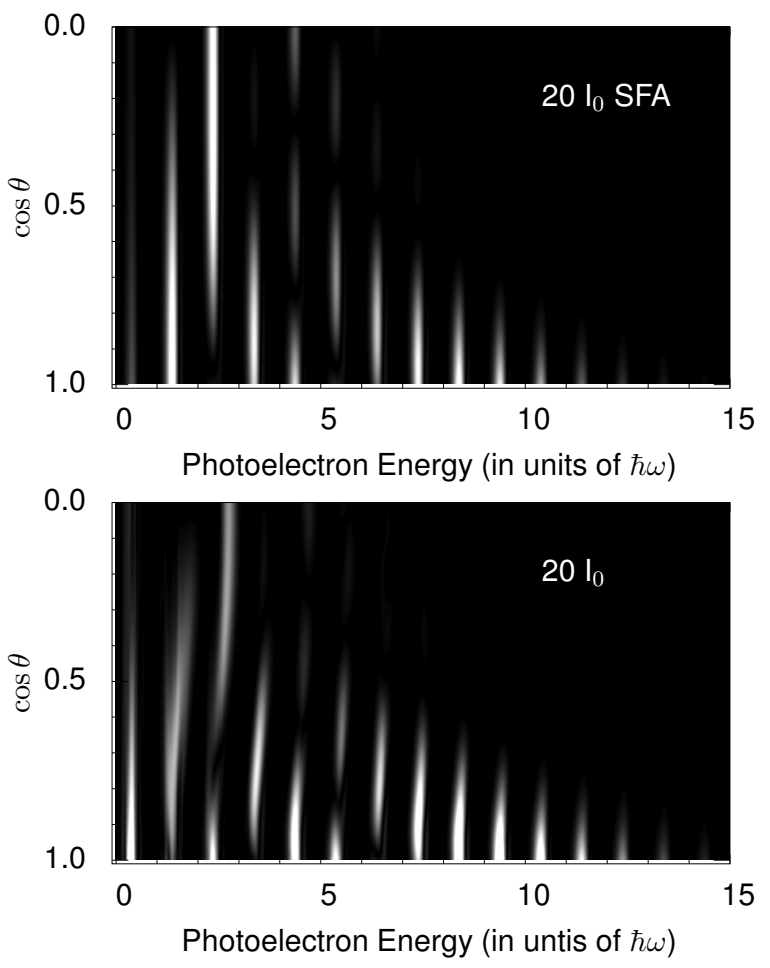

FIG. 2. Photoelectron energy-angle distribution of $\mathrm{H}$ atoms ionized by the XUV light in an IR field calculated by the SFA (upper panel, replot of Fig. 1) and TDSE (lower panel) for the IR intensity of $20 I_{0}$.

\section{B. IR laser intensity}

Since the IR intensity is a key quantity in the strong field material interaction, we first investigate how the intensity affects the angular distribution. For such a purpose, we analyze the angular distributions from no IR laser to a relative strong one at $50 I_{0}$ with a $10^{12} \mathrm{~W} / \mathrm{cm}^{2}$ intensity step systematically as shown in Ref [29. We find that with a weak IR intensity $\left(<10^{13} \mathrm{~W} / \mathrm{cm}^{2}\right)$, there is no visible bend as shown in Fig. 3 , a typical example. As the IR intensity increases, the visible bending appears at $1.5 \times 10^{13} \mathrm{~W} / \mathrm{cm}^{2}$ and the bending persists to high IR intensities. This means that both the Coulomb effect and IR laser field contribute to the bending. The incline of low energy sidebands increases as the IR intensity increases [29].

\section{XUV photon energy}

Since the Coulomb interaction is important for low energy electrons, if we use a high energy XUV photon, does the bending still exist? To answer the question, we increase the XUV photon energy to $30 \mathrm{eV}$ as shown in Fig. 4 and find that the sidebands still bend to the high energy for large angles but the incline is smaller than the case for $20 \mathrm{eV}$ with the same IR intensity. Since the vis-

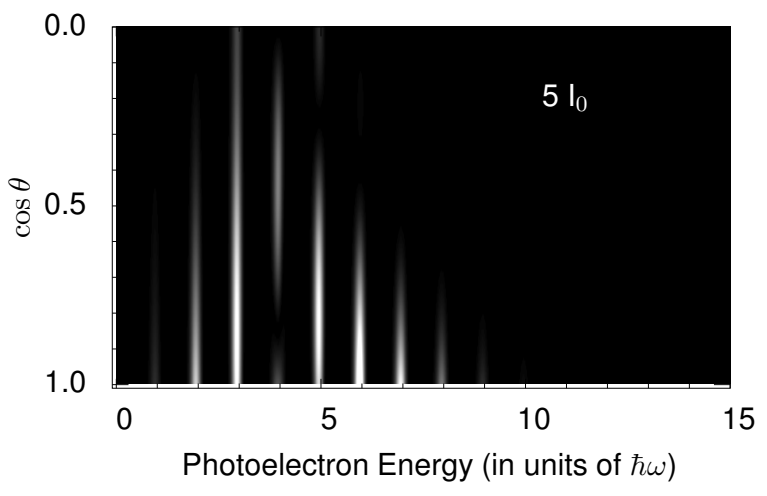

FIG. 3. Photoelectron energy-angle distribution of $\mathrm{H}$ atoms ionized by the XUV light in a weak IR intensity of $5 I_{0}$.

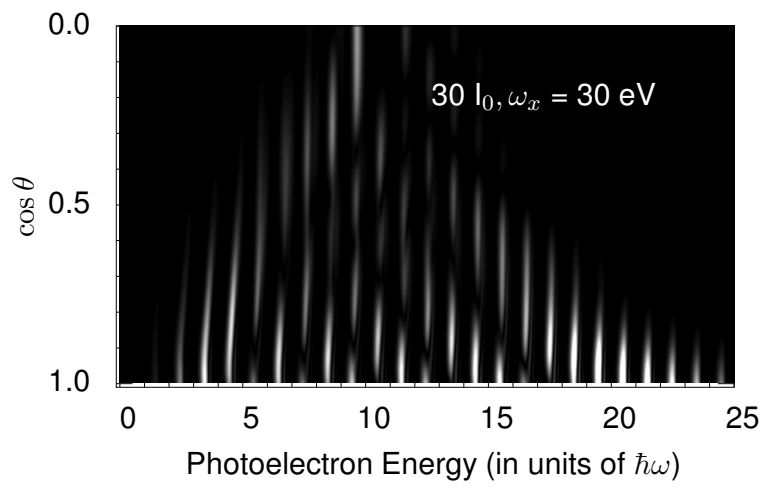

FIG. 4. Photoelectron energy-angle distribution of $\mathrm{H}$ atoms ionized by a $30 \mathrm{eV}$ XUV light in an IR laser with the intensity of $30 I_{0}$.

ible bending starts at a high IR intensity for high XUV photon energy so we show the results with a high IR intensity of $30 I_{0}$ at which the sidebands spread broader and extend to the ionization threshold.

\section{IR pulse duration}

The atomic photoionization by an XUV light in an IR field can be explained by the Floquet theorem [8, 11] and the sideband should be iso-energy distribution or straight lines in the energy-angle plots if the IR pulse is infinite long. If we increase the IR pulse duration to $60 \mathrm{fs}$, the bending does disappear gradually as show in Fig. 55apart from a few lines in the very low energies, the observation is in consistent with the prediction by the Floquet theorem. From this comparison, we conclude that the bending can be further originated to the phase of the photoelectron obtained in one IR cycle in a Coulomb field. The phase also depends on the photoelectron moving direction. For a very long IR pulse, the obtained phase will be repeated every IR cycle, while for a short pulse, such a phase differs slightly in each IR cycle, so the final photoelectron energy depends on the electron moving direction, which results in the bending. The systematic 


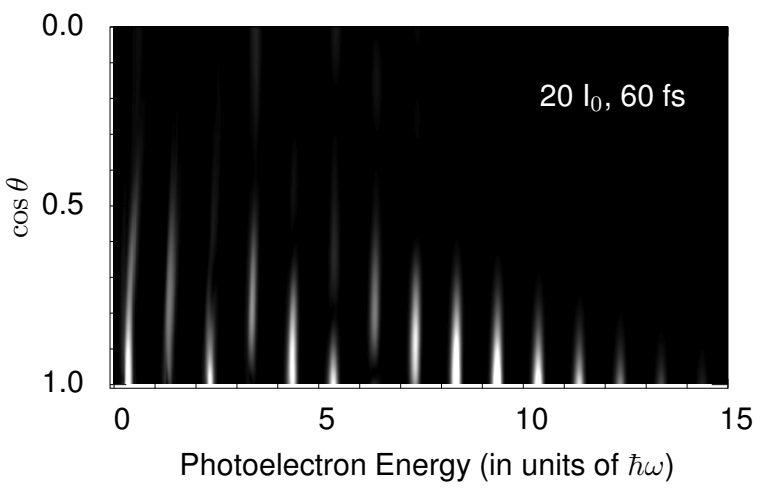

FIG. 5. Photoelectron energy-angle distribution of $\mathrm{H}$ atoms ionized by the XUV light in the IR field with the pulse duration of $60 \mathrm{fs}$.

evolution of the photoelectron energy-angle distribution as a function of the pulse durations from 20 fs to $120 \mathrm{fs}$ with a 2 fs step can be found in Ref. [30.

\section{E. Time-delay}

Figure 6 shows the energy-angle distribution for the IR pulse arrives 16 fs before the XUV pulse. The bending still exists but the sidebands bend to the lower energy for large angles, which differ from the results when the two pulses overlap with each other. The systematic evolution of the photoelectron energy-angle distribution as a function of the time-delay from -25 fs to $25 \mathrm{fs}$ with a $1 \mathrm{fs}$ step can be found in Ref. 31.

As we see in Eq. (5) that the IR vector potential also depends on the CEP, but the TDSE results show that the bending is insensitive to the CEP. We give the conclusion without presenting the results here anymore.

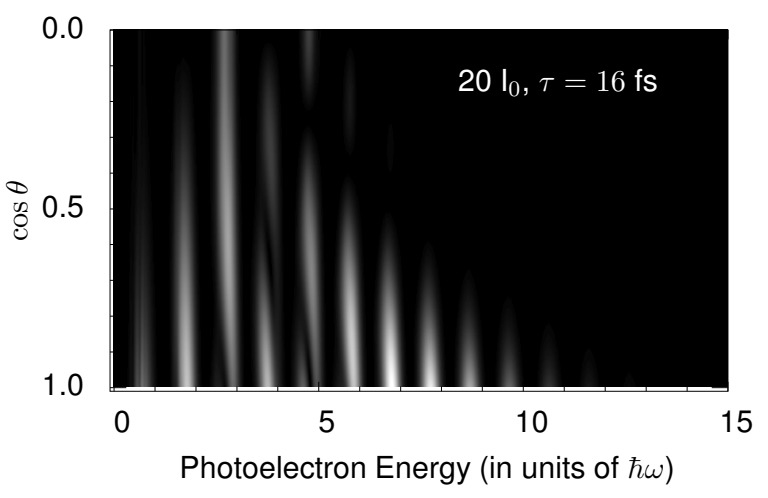

FIG. 6. Photoelectron energy-angle distribution of $\mathrm{H}$ atoms ionized by the XUV light in the IR field with 16 fs time delay.

\section{F. Physical origin}

Comparing the results of the SFA and TDSE, we can identify that the Coulomb interaction results in the abnormality. We may ask why the sideband energy depends on the outgoing photoelectron direction. This is related to the phase of the photoelectron gained in the IR field after the electron is ejected out from the parent atom by the XUV light. The phase is expressed as

$$
\delta_{p}(t)=\int_{t}^{\infty} H\left(t^{\prime}\right) d t^{\prime},
$$

as shown in Eq. (1). If the IR pulse is infinity long, the phase is a periodic function of time and the sidebands are always straight lines on the energy-angle plots which does not rely on whether the Coulomb interaction is considered or not. If the IR pulse is not infinite long, the phase obtained within each IR cycle differs slightly since the detailed IR electric field changes cycle by cycle, so the sidebands are almost on the iso-energy rings if the Coulomb interaction is ignored. If the Coulomb interaction is considered, the phase of the photoelectron moving in a Coulomb field within one IR cycle depends on the electron trajectory or the moving direction. Since the Coulomb effect is important for low energy electrons, the inclines are larger for lower energy sidebands. The detailed dependence could be complex, but the physical explanation should be right from the above discussion. We also calculated the photoelectron angular distribution by replacing the XUV light from free-electron laser with the APT, the bending still exists.

This observation is related to the "ionization surprise", which was observed in the above-threshold ionization (ATI) in experiments [32, 33] and attributed the surprise to the photoelectron Coulomb interaction [34 37. If we carefully looked at the two-dimensional momentum distributions of the photoelectron in intense midIR laser fields [38 41], indeed the photoelectrons are not iso-energy distributed, especially on the low energy side. Therefore, even for the atomic ATI spectra in an intense IR field, the SFA does not work very well for the angular distribution of low energy ATIs.

The mechanism of the bending of photoelectron angular distribution for an IR assisted photoionization in a long XUV pulse is different from the one for an IR assisted SAP photoionization. For the SAP, the energy spreads broadly and covers several IR photon energies so there are no clearly sidebands separated by one IR photon energy. The SAP can be used for the streaking experiments [42, 45].

\section{SUMMARY AND CONCLUSION}

In this work, we have investigated the bending of photoelectron angular distribution of atoms ionized by an XUV light assisted by a moderate intense IR laser field. The bending is attributed to the photoelectron nucleus 
Coulomb interaction since the angular distribution returns to the straight line in the energy-angle plots if the Coulomb interaction is ignored in the simulation. The bending depends on the XUV photon energy, IR intensity, IR pulse duration as well as the time-delay between the two pulses. Therefore, it can be used to calibrate or obtain the laser parameters by comparing measurements with simulations. Note that the focal volume averaging is not important since the focal size of XUV sources, like APT, is much narrower than the focal size of IR fields. The effect is also not sensitive to the atomic species since the XUV creates a quasi-free electron and the IR electric field drifts the quasi-free electron moving in a combined IR electric field and nuclear Coulomb interaction.

\section{ACKNOWLEDGMENTS}

This work was supported by a Grants-in-Aid for Scientific Research (JP16K05495) from the Japan Society for the Promotion of Science. The TDSE calculations were performed using Oakforest-Pacs at Center for Computational Sciences, University of Tsukuba.
[1] X. F. Li, A. L'Huillier, M. Ferray, L. A. Lompre, and G. Mainfray, Phys. Rev. A 39, 5751 (1989)

[2] A. L'Huillier, K. J. Schafer, and K. C. Kulander, J. Phys. B: At. Mol. Opt. Phys. 24, 3315 (1991)

[3] V. Véniard, R. Taïeb, and A. Maquet, Phys. Rev. Lett. 74, 4161 (1995)

[4] T. E. Glover, R. W. Schoenlein, A. H. Chin, and C. V. Shank, Phys. Rev. Lett. 76, 2468 (1996)

[5] E. S. Toma, H. G. Muller, P. M. Paul, P. Breger, M. Cheret, P. Agostini, C. Le Blanc, G. Mullot, and G. Cheriaux, Phys. Rev. A 62, 061801 (2000).

[6] P. Johnsson, J. Mauritsson, T. Remetter, A. L'Huillier, and K. J. Schafer, Phys. Rev. Lett. 99, 233001 (2007).

[7] P. Ranitovic, X. M. Tong, B. Gramkow, S. De, B. DePaola, K. P. Singh, W. Cao, M. Magrakvelidze, D. Ray, I. Bocharova, H. Mashiko, A. Sandhu, E. Gagnon, M. M. Murnane, H. Kapteyn, I. Litvinyuk, and C. L. Cocke, New J. Phys. 12, 013008 (2010).

[8] S. I. Chu and D. A. Telnov, Phys. Rep. 390, 1 (2004).

[9] X. M. Tong, P. Ranitovic, C. L. Cocke, and N. Toshima, Phys. Rev. A 81, 021404 (2010).

[10] X.-M. Tong and N. Toshima, Phys. Rev. A 81, 043429 (2010)

[11] X. M. Tong and N. Toshima, Phys. Rev. A 81, 063403 (2010)

[12] P. Ranitovic, X. M. Tong, C. W. Hogle, X. Zhou, Y. Liu, N. Toshima, M. M. Murnane, and H. C. Kapteyn, Phys. Rev. Lett. 106, 193008 (2011)

[13] P. Antoine, A. L'Huillier, and M. Lewenstein, Phys. Rev. Lett. 77, 1234 (1996)

[14] P. M. Paul, E. S. Toma, P. Breger, G. Mullot, F. Auge, P. Balcou, H. G. Muller, and P. Agostini, Science 292, 1689 (2001)

[15] Z. Chang, Phys. Rev. A 70, 043802 (2004)

[16] G. Sansone, E. Benedetti, F. Calegari, C. Vozzi, L. Avaldi, R. Flammini, L. Poletto, P. Villoresi, C. Altucci, R. Velotta, S. Stagira, S. De Silvestri, and M. Nisoli, Science 314, 443 (2006).

[17] Z. Huang and K.-J. Kim, Phys. Rev. ST Accel. Beams 10 (2007), 10.1103/physrevstab.10.034801

[18] A. Cionga, V. Florescu, A. Maquet, and R. Taeb, Phys. Rev. A 47, 1830 (1993)

[19] A. K. Kazansky, I. P. Sazhina, and N. M. Kabachnik, Phys. Rev. A 82, 033420 (2010).

[20] S. Minemoto, H. Shimada, K. Komatsu, W. Komatsubara, T. Majima, T. Mizuno, S. Owada, H. Sakai, T. To- gashi, S. Yoshida, M. Yabashi, and A. Yagishita, J. Phys. B: At. Mol. Opt. Phys. 51, 075601 (2018).

[21] J. Hummert, M. Kubin, S. D. López, M. J. Vrakking, O. Kornilov, and D. G. Arbó, arXiv preprint arXiv:1808.04639 (2018).

[22] M. Lewenstein, P. Balcou, M. Y. Ivanov, A. L'Huillier, and P. B. Corkum, Phys. Rev. A 49, 2117 (1994)

[23] A. L'Huillier, M. Lewenstein, P. Salieres, P. Balcoui, J. Larson, and C. G. Wahlstrom, Phys. Rev. A 48, R3433 (1993)

[24] A. Becker and F. H. M. Faisal, J. Phys. B: At. Mol. Opt. Phys. 38, R1 (2005)

[25] A.-T. Le, H. Wei, C. Jin, and C. D. Lin, J. Phys. B: At. Mol. Opt. Phys. 49, 053001 (2016)

[26] X.-M. Tong and S.-I. Chu, Chem. Phys. 217, 119 (1997).

[27] X. M. Tong, K. Hino, and N. Toshima, Phys. Rev. A 74, 031405 (2006)

[28] X. M. Tong, S. Watahiki, K. Hino, and N. Toshima, Phys. Rev. Lett. 99, 093001 (2007).

[29] S. S. M. at mov01 for the photoelectron angular distibution of hydrogen atoms ionized by an XUV light in the IR fields with different IR intensities, .

[30] S. S. M. at mov02 for photoelectron angular distibution of hydrogen atoms ionized by an XUV light in the IR fields with different IR pulse durations., .

[31] S. S. M. at mov03 for the photoelectron angular distibution of hydrogen atoms ionized by an XUV light in the IR fields with different time-delays between the two pulsese., .

[32] C. I. Blaga, F. Catoire, P. Colosimo, G. G. Paulus, H. G. Muller, P. Agostini, and L. F. DiMauro, Nature phyics 5, 335 (2009).

[33] W. Quan, Z. Lin, M. Wu, H. Kang, H. Liu, X. Liu, J. Chen, J. Liu, X. T. He, S. G. Chen, H. Xiong, L. Guo, H. Xu, Y. Fu, Y. Cheng, and Z. Z. Xu, Phys. Rev. Lett. 103, 093001 (2009)

[34] F. H. M. Faisal, Nature, Phys. 5, 319 (2009).

[35] C. Liu and K. Z. Hatsagortsyan, Phys. Rev. Lett. 105, $113003(2010)$

[36] T.-M. Yan, S. V. Popruzhenko, M. J. J. Vrakking, and D. Bauer, Phys. Rev. Lett. 105, 253002 (2010).

[37] L. Guo, S. S. Han, X. Liu, Y. Cheng, Z. Z. Xu, J. Fan, J. Chen, S. G. Chen, W. Becker, C. I. Blaga, A. D. DiChiara, E. Sistrunk, P. Agostini, and L. F. DiMauro, Phys. Rev. Lett. 110, 013001 (2013) 
[38] Y. Huismans, A. Rouzée, A. Gijsbertsen, J. H. Jungmann, A. S. Smolkowska, P. S. W. M. Logman, F. Lépine, C. Cauchy, S. Zamith, T. Marchenko, J. M. Bakker, G. Berden, B. Redlich, A. F. G. van der Meer, H. G. Muller, W. Vermin, K. J. Schafer, M. Spanner, M. Y. Ivanov, O. Smirnova, D. Bauer, S. V. Popruzhenko, and M. J. J. Vrakking, Science 331, 61 (2011).

[39] D. D. Hickstein, P. Ranitovic, S. Witte, X.-M. Tong, Y. Huismans, P. Arpin, X. Zhou, K. E. Keister, C. W. Hogle, B. Zhang, C. Ding, P. Johnsson, N. Toshima, M. J. J. Vrakking, M. M. Murnane, and H. C. Kapteyn, Phys. Rev. Lett. 109, 073004 (2012).

[40] C. Lemell, K. I. Dimitriou, X.-M. Tong, S. Nagele, D. V. Kartashov, J. Burgdörfer, and S. Gräfe, Phys. Rev. A 85, $011403(2012)$.

[41] X.-M. Tong, P. Ranitovic, D. D. Hickstein, M. M. Murnane, H. C. Kapteyn, and N. Toshima, Phys. Rev. A 88,
$013410(2013)$

[42] J. Itatani, F. Quéré, G. L. Yudin, M. Y. Ivanov, F. Krausz, and P. B. Corkum, Phys. Rev. Lett. 88, 173903 (2002)

[43] E. Goulielmakis, M. Uiberacker, R. Kienberger, A. Baltuska, V. Yakovlev, A. Scrinzi, T. Westerwalbesloh, U. Kleineberg, U. Heinzmann, M. Drescher, and F. Krausz, Science 305, 1267 (2004).

[44] M.-H. Xu, L.-Y. Peng, Z. Zhang, Q. Gong, X.-M. Tong, E. A. Pronin, and A. F. Starace, Phys. Rev. Lett. 107, 183001 (2011)

[45] M. Kübel, Z. Dube, A. Y. Naumov, M. Spanner, G. G. Paulus, M. F. Kling, D. M. Villeneuve, P. B. Corkum, and A. Staudte, Phys. Rev. Lett. 119, 183201 (2017). 